Benchmarking doubles-corrected random-phase approximation methods for frequency dependent polarizabilities: aromatic molecules calculated at the RPA, HRPA, RPA(D), HRPA(D) and SOPPA levels

Jørgensen, Maria West; Sauer, Stephan P. A.

Published in:

Journal of Chemical Physics

DOI:

$10.1063 / 5.0011195$

Publication date:

2020

Document version

Peer reviewed version

Document license:

CC BY-NC

Citation for published version (APA):

Jørgensen, M. W., \& Sauer, S. P. A. (2020). Benchmarking doubles-corrected random-phase approximation methods for frequency dependent polarizabilities: aromatic molecules calculated at the RPA, HRPA, RPA(D), HRPA(D) and SOPPA levels. Journal of Chemical Physics, 152, [234101]. https://doi.org/10.1063/5.0011195 


\title{
Benchmarking doubles-corrected random-phase approximation methods for frequency dependent polarizabilities: aromatic molecules calculated at the RPA, HRPA, RPA(D), HRPA(D) and SOPPA levels
}

\author{
Maria W. Jørgensen ${ }^{1}$ and Stephan P. A. Sauer ${ }^{1, \text { a) }}$ \\ Department of Chemistry, University of Copenhagen, Copenhagen $\varnothing$, \\ Denmark
}

(Dated: 21 May 2020)

The performance of different polarization propagator methods, RPA, RPA(D), HRPA, HRPA(D) and SOPPA have been tested against CC3 values for both static and dynamic polarizabilities. The test set consists of 14 (hetero-)aromatic medium size organic molecules, mostly with a high degree of symmetry. The benchmark of the methods remarkably reveals that RPA and $\operatorname{HRPA}(\mathrm{D})$ yield results comparable with the CC3 values and that they outperform SOPPA for these molecules. For a subset of the molecules a comparison could be made to experimental values. The comparison for static polarizabilities proves that RPA and HRPA(D) as well as RPA(D) reproduce experimental values to a satisfying precision, whereas the SOPPA method compared to these two methods appears to perform only adequately. An investigation of the performance of Sadlej's polarized triple zeta basis set against Dunning's augcc-pVTZ basis set was also carried out. It is found, that in contrast to other methods, Sadlej's basis set did not perform sufficiently compared to the larger aug-cc-pVTZ basis set for the RPA based methods.

a) sauer@kiku.dk 


\section{INTRODUCTION}

Coupled cluster methods like CCSD and in particular methods including also contributions from triple excitations like CCSDT, $\operatorname{CCSD}(\mathrm{T})$ or CC3 have time and time again been shown to give highly accurate results for molecular properties like e.g. polarizabilities $^{1-3}$. However, their high computational demand limits the usage of these, seemingly ideal, methods. Thence, there has been a great focus on developing different methods, which give the same, or slightly lower, accuracy but at lower cost. In a recent study ${ }^{4}$ CC3 values for the static and dynamic polarizabilities of medium size (hetero-)aromatic molecules were presented. Using these CC3 results as reference values, the performance of different second order methods such as SOPPA, SOPPA(CC2), SOPPA(CCSD), CC2 and CCSD were investigated. In the present work the scope of investigated methods is extended to include two precursor methods to SOPPA, random phase approximation (RPA) and higher-order random phase approximation (HRPA), as well as two approximations to SOPPA, the random phase approximation with doubles corrections ${ }^{5}, \mathrm{RPA}(\mathrm{D})$, as well as the recently proposed higher-order random phase approximation with doubles corrections, $\operatorname{HRPA}(\mathrm{D})^{6,7}$.

The random phase approximation, RPA, is a rather old method in quantum chemistry and is also known as time-dependent Hartree-Fock, TDHF, which might reveal its old age. Back in 1968 the RPA was re-derived using the equations of motion method ${ }^{8}$. Although this was a more efficient way, it also showed which parts are neglected in this method. Shortly after a higher-order random phase approximation, HRPA, was derived ${ }^{9}$, also from the equations of motion method. Unlike RPA, which is only correct through first-order, HRPA includes contributions of second-order in the A and B matrices. The HRPA method was in this early study found to improve on some of the deficiencies of RPA. However, it is also known to overestimate excitation energies as shown again in the recent study by Haase et $a .^{7}$.

Both these methods, RPA and HRPA, only contain single (de-)excitation operators, while including also double (de-)excitation operators yields a method correct through second-order, the SOPPA method ${ }^{10-12}$. As was seen in the previous study ${ }^{4}$, SOPPA predicts polarizabilities rather close to the $\mathrm{CC} 3$ results and is able to reproduce experimental, static and dynamic, isotropic polarizabilities with good precision. For other properties like NMR spin-spin coupling constants it is known that the extra electron correlation in SOPPA, which lacks in RPA, 
can be of high importance and leaves the method unaffected by triplet-instabilities ${ }^{6,13-16}$. A challenge with the SOPPA method is the dimensions of the matrices, which makes this method more demanding than density functional theory methods.

The quality of the SOPPA method created an interest in finding a method of the same accuracy but reduced computational demands. This led Christiansen et. al. ${ }^{5}$ in 1997 to present an approximation to SOPPA. This method, reminding of the RPA, adds contributions of second-order in a non-iterative manner. The double corrections, being added non-iteratively, lead to a significantly lower computational cost than for the original SOPPA method, as no iterative solutions of linear equations above the RPA level are needed. Christiansen tested this method, known as RPA(D), for excitation energies of small molecules and found that it performed similarly to SOPPA and CC2 when compared to $\mathrm{FCI}^{5}$, while others employed it in the calculation of excitation energies of azo-dyes or polyaromatic hydrocarbons ${ }^{17-20}$. The recent study of Haase et $a .^{7}$ also describes a higher-order doubles-corrected method, HRPA(D). For the Thiel benchmark set of vertical excitation energies ${ }^{21-23}$, it was found that both doubles-corrected methods performed significantly closer to the SOPPA method than RPA and HRPA. However, though the mean and standard deviations from CC3 results are smaller compared to RPA and HRPA, the standard deviation is still twice that of SOPPA. RPA(D), as in Christiansen's study, was found to be an improvement of RPA but when RPA suffered from triplet instabilities, RPA(D) would be drastically affected. On the other hand, HRPA(D) did not seem affected by triplet instabilities at all. Two recent studies ${ }^{6,24}$ calculated indirect nuclear spin-spin coupling constants using the same methods and found the same tendencies. It was pointed out that the improvement of the doubles-corrected methods is more pronounced for RPA than for HRPA. HRPA(D), though performing comparable with SOPPA, still needs between $30 \%$ and $90 \%$ of the calculation time of SOPPA. Therefore, the method, at times, is still not performing fast enough. The RPA(D), on the other hand, performed slightly less close to SOPPA but the computational time was down to $25 \%$, making this method comparatively fast with only a slight loss of precision ${ }^{6,7}$. In the present study the performance of both these doubles-corrected methods is for the first time investigated for static and frequency-dependent isotropic polarizabilities.

One should mention here that there exist also a related but alternative approach for the calculation of excitation energies and response functions, which is based on a Møller-Plesset perturbation theory expansion of the polarization propagator, called the Algebraic Dia- 
grammatic Construction (ADC). ${ }^{25}$ The first-order $\mathrm{ADC}^{25}$ model is analogous to RPA, the similarities of the ADC and RPA methods is elucidated as the RPA has before been reformulated using the algebraic-diagrammatic approximation ${ }^{26}$. In the same manner, $\operatorname{ADC}(2)^{27}$ is of second-order and can thus be compared to SOPPA as e.g. done for frequency dependent polarizabilities of small molecules. ${ }^{28}$ Although expressions for the matrix elements of a third-order polarization propagator have been derived, the method has not been fully implemented and not yet applied in studies. ${ }^{29}$ On the other hand, the theory of a third-order ADC model, $\mathrm{ADC}(3)^{30,31}$, is fully described and a so-called incomplete third-order ADC model which is theoretically in between second and third order, the $\operatorname{ADC}(3 / 2)^{32}$ model, has been developed. A benchmark study ${ }^{33}$ compared ADC(3/2) to CCSD for electron correlation effects on static polarizabilities and $C_{6}$ dispersion coefficients and found the methods to perform comparatively. The results of the SOPPA method have several times been shown to improve, if the correlation coefficients in the wavefunctions are replaced by coupled cluster amplitudes ${ }^{4,15,16,34-39}$. This inspired Hodecker et. al. to implement also coupled cluster intermediate models of ADC, the CCD-ADC $(2)^{40}$ and CCSD-ADC $(2)^{41}$ models. In the latter study, polarizabilities of aromatic organic molecules were tested for benzene and pyridine, which have previously been shown to be difficult cases for the ADC model. This study found the ADC method to consistently improve for isotropic polarizabilities but also for anisotropic polarizabilities with the increasing level of coupled cluster theory applied as had been seen previously for the corresponding SOPPA methods. ${ }^{15}$

\section{COMPUTATIONAL}

Figure 1 shows the 14 (hetero-)aromatic medium-sized molecules used in this study and their names. The geometries for benzene, furan, pyrrole, imidazole, pyridine, pyrimidine, pyrazine and pyridazine were taken from a previous study ${ }^{22}$. Oxazole, thiazole, thiophene, phosphole, phenol and benzonitrile were optimized in the previous study ${ }^{4}$. All geometries were thus previously optimized at the MP2/6-31G(d) level. For this study the aug-cc$\mathrm{pVTZ}^{42-44}$ basis set has been used as well as Sadlej's polarized triple zeta basis set ${ }^{45,46}$. Polarizabilities with the $\mathrm{RPA}^{47}$ and $\mathrm{HRPA}^{48}$ methods were calculated in DALTON ${ }^{49}$, whereas the $\operatorname{RPA}(\mathrm{D})^{50}$ and $\operatorname{HRPA}(\mathrm{D})^{7}$ polarizabilities were calculated in a local development version of DALTON. The polarizabilities calculated at the SOPPA ${ }^{51}$ level are taken from the 
previous study ${ }^{4}$. All methods use an unrelaxed approach to ensure the pole-structure of the polarizability. The polarizabilities are isotropic polarizabilities found from the trace of the polarizability tensor:

$$
\alpha=\frac{1}{3}\left(\alpha_{x x}+\alpha_{y y}+\alpha_{z z}\right)
$$

The benchmark of the methods is carried out for both static and dynamic polarizabilities with frequencies $0.072003 \mathrm{au}(632 \mathrm{~nm})$ and $0.093215 \mathrm{au}(488.8 \mathrm{~nm})$.

A component of the frequency dependent polarizability is in this study calculated as the linear response function or polarization propagator of the corresponding components of the dipole moment operator:

$$
\alpha_{\alpha \beta}(\omega)=-\left\langle\left\langle\hat{\mu}_{\alpha} ; \hat{\mu}_{\beta}\right\rangle\right\rangle_{\omega}
$$

At the RPA level the polarization propagator is given as:

$$
\langle\langle\hat{P} ; \hat{O}\rangle\rangle_{\omega}^{R P A}=\left(\begin{array}{ll}
{ }^{e} \boldsymbol{P}^{(0)} & { }^{d} \boldsymbol{P}^{(0)}
\end{array}\right)\left(\begin{array}{c}
{ }^{e} \boldsymbol{X}^{R P A} \\
{ }^{d} \boldsymbol{X}^{R P A}
\end{array}\right)
$$

When the operators $\hat{P}$ and $\hat{O}$ are one of the three components of the dipole moment operator, the response function will yield a component of the polarizability. The RPA solution vector is obtained from the RPA linear equations:

$$
\left(\begin{array}{cc}
\omega \mathbf{1}-\boldsymbol{A}^{(0,1)} & -\boldsymbol{B}^{(1)} \\
-\boldsymbol{B}^{(1)} & -\omega \mathbf{1}-\boldsymbol{A}^{(0,1)}
\end{array}\right)\left(\begin{array}{c}
{ }^{e} \boldsymbol{X}^{R P A} \\
{ }^{d} \boldsymbol{X}^{R P A}
\end{array}\right)=\left(\begin{array}{c}
{ }^{e} \boldsymbol{O}^{(0)} \\
{ }^{d} \boldsymbol{O}^{(0)}
\end{array}\right)
$$

In RPA(D) the polarization propagator consist of two terms ${ }^{6}$ :

$$
\langle\langle\hat{P} ; \hat{O}\rangle\rangle_{\omega}^{R P A(D)}=\langle\langle\hat{P} ; \hat{O}\rangle\rangle_{\omega}^{R P A(D), S}+\langle\langle\hat{P} ; \hat{O}\rangle\rangle_{\omega}^{R P A(D), D}
$$

where the first consists of contributions from single excitations:

$$
\begin{aligned}
& \langle\langle\hat{P} ; \hat{O}\rangle\rangle_{\omega}^{R P A(D), S}
\end{aligned}
$$

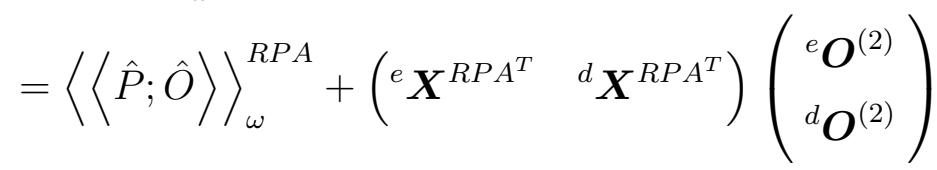

$$
\begin{aligned}
& +\left(\begin{array}{cc}
{ }^{e} \boldsymbol{X}^{R P A^{T}} & { }^{d} \boldsymbol{X}^{R P A^{T}}
\end{array}\right)\left(\begin{array}{cc}
\boldsymbol{A}^{(2)}-\omega \boldsymbol{\Sigma}^{(2)} & \boldsymbol{B}^{(2)} \\
\boldsymbol{B}^{(2)} & \omega \boldsymbol{\Sigma}^{(2)}+\boldsymbol{A}^{(2)}
\end{array}\right)\left(\begin{array}{c}
{ }^{e} \boldsymbol{X}^{R P A} \\
{ }^{d} \boldsymbol{X}^{R P A}
\end{array}\right) \\
& +\left(\begin{array}{ll}
{ }^{e} \boldsymbol{P}^{(2)} & { }^{d} \boldsymbol{P}^{(2)}
\end{array}\right)\left(\begin{array}{c}
{ }^{e} \boldsymbol{X}^{R P A} \\
{ }^{d} \boldsymbol{X}^{R P A}
\end{array}\right)
\end{aligned}
$$


and the second of contributions from double excitations:

$$
\begin{aligned}
& \langle\langle\hat{P} ; \hat{O}\rangle\rangle_{\omega}^{R P A(D), D} \\
& =-\left\{\left(\begin{array}{ll}
{ }^{e} \boldsymbol{\Pi}^{(1)} & { }^{d} \boldsymbol{\Pi}^{(1)}
\end{array}\right)+\left(\begin{array}{ll}
{ }^{e} \boldsymbol{X}^{R P A^{T}} & { }^{d} \boldsymbol{X}^{R P A^{T}}
\end{array}\right)\left(\begin{array}{cc}
\widetilde{\boldsymbol{C}}^{(1)} & 0 \\
0 & \widetilde{\boldsymbol{C}}^{(1)}
\end{array}\right)\right\} \\
& \times\left(\begin{array}{cc}
\boldsymbol{D}^{(0)}-\omega \mathbf{1} & 0 \\
0 & \boldsymbol{D}^{(0)}+\omega \mathbf{1}
\end{array}\right)^{-1}\left\{\left(\begin{array}{c}
{ }^{e} \boldsymbol{\Omega}^{(1)} \\
{ }^{d} \boldsymbol{\Omega}^{(1)}
\end{array}\right)+\left(\begin{array}{cc}
\boldsymbol{C}^{(1)} & 0 \\
0 & \boldsymbol{C}^{(1)}
\end{array}\right)\left(\begin{array}{l}
{ }^{e} \boldsymbol{X}^{R P A} \\
{ }^{d} \boldsymbol{X}^{R P A}
\end{array}\right)\right\}
\end{aligned}
$$

In HRPA the response function is defined as:

$$
\langle\langle\hat{P} ; \hat{O}\rangle\rangle_{\omega}^{H R P A}=\left(\begin{array}{ll}
{ }^{e} \boldsymbol{P}^{(0,2)} & { }^{d} \boldsymbol{P}^{(0,2)}
\end{array}\right)\left(\begin{array}{c}
{ }^{e} \boldsymbol{X}^{H R P A} \\
{ }^{d} \boldsymbol{X}^{H R P A}
\end{array}\right)
$$

where the HRPA solution vector is obtained from the HRPA linear equations:

$$
\left(\begin{array}{cc}
\omega\left(\mathbf{1}+\boldsymbol{\Sigma}^{(2)}\right)-\boldsymbol{A}^{(0,1,2)} & -\mathbf{B}^{(1,2)} \\
-\boldsymbol{B}^{(1,2)} & -\omega\left(\mathbf{1}+\boldsymbol{\Sigma}^{(2)}\right)-\boldsymbol{A}^{(0,1,2)}
\end{array}\right)\left(\begin{array}{c}
{ }^{e} \boldsymbol{X}^{H R P A} \\
{ }^{d} \boldsymbol{X}^{H R P A}
\end{array}\right)=\left(\begin{array}{c}
{ }^{e} \boldsymbol{O}^{(0,2)} \\
{ }^{d} \boldsymbol{O}^{(0,2)}
\end{array}\right)
$$

In HRPA(D) the HRPA polarization propagator is then extended with a term from double excitations $^{6}$ :

$$
\begin{aligned}
& \langle\langle\hat{P} ; \hat{O}\rangle\rangle_{\omega}^{H R P A(D)}=\langle\langle\hat{P} ; \hat{O}\rangle\rangle_{\omega}^{H R P A}+\langle\langle\hat{P} ; \hat{O}\rangle\rangle_{\omega}^{H R P A(D), D} \\
& \langle\langle\hat{P} ; \hat{O}\rangle\rangle_{\omega}^{H R P A(D), D} \\
& =-\left\{\left(\begin{array}{ll}
{ }^{e} \boldsymbol{\Pi}^{(1)} & { }^{d} \boldsymbol{\Pi}^{(1)}
\end{array}\right)+\left(\begin{array}{ll}
{ }^{e} \boldsymbol{X}^{H R P A^{T}} & { }^{d} \boldsymbol{X}^{H R P A^{T}}
\end{array}\right)\left(\begin{array}{cc}
\widetilde{\boldsymbol{C}}^{(1)} & 0 \\
0 & \widetilde{\boldsymbol{C}}^{(1)}
\end{array}\right)\right\} \\
& \times\left(\begin{array}{cc}
\boldsymbol{D}^{(0)}-\omega \mathbf{1} & 0 \\
0 & \boldsymbol{D}^{(0)}+\omega \mathbf{1}
\end{array}\right)^{-1}\left\{\left(\begin{array}{c}
{ }^{e} \boldsymbol{\Omega}^{(1)} \\
{ }^{d} \boldsymbol{\Omega}^{(1)}
\end{array}\right)+\left(\begin{array}{cc}
\boldsymbol{C}^{(1)} & 0 \\
0 & \boldsymbol{C}^{(1)}
\end{array}\right)\left(\begin{array}{l}
{ }^{e} \boldsymbol{X}^{H R P A} \\
{ }^{d} \boldsymbol{X}^{H R P A}
\end{array}\right)\right\}
\end{aligned}
$$

The elements of the individual matrices and vectors are defined as:

$$
\begin{aligned}
A_{a i, b j}^{(0,1,2)} & =\left\langle\Phi_{0}^{M P}\left|\left[q_{a i}\left[\hat{H}, q_{b j}^{\dagger}\right]\right]\right| \Phi_{0}^{M P}\right\rangle^{(0,1,2)} \\
B_{a i, b j}^{(1,2)} & =\left\langle\Phi_{0}^{M P}\left|\left[q_{a i}\left[\hat{H}, q_{b j}\right]\right]\right| \Phi_{0}^{M P}\right\rangle^{(1,2)} \\
D_{a i b j, c k d l}^{(0)} & =\left\langle\Phi_{0}^{H F}\left|\left[q_{a i} q_{b j},\left[\hat{H}, q_{c k}^{\dagger} q_{d l}^{\dagger}\right]\right]\right| \Phi_{0}^{H F}\right\rangle^{(0)}
\end{aligned}
$$




$$
\begin{aligned}
& C_{a i b j, c k}^{(1)}=\left\langle\Phi_{0}^{H F}\left|\left[q_{a i} q_{b j},\left[\hat{H}, q_{c k}^{\dagger}\right]\right]\right| \Phi_{0}^{H F}\right\rangle^{(1)} \\
& \widetilde{C}_{c k, a i b j}^{(1)}=\left\langle\Phi_{0}^{H F}\left|\left[q_{c k},\left[\hat{H}, q_{a i}^{\dagger} q_{b j}^{\dagger}\right]\right]\right| \Phi_{0}^{H F}\right\rangle^{(1)} \\
& \Sigma_{a i, b j}^{(0,2)}=\left\langle\Phi_{0}^{M P}\left|\left[q_{a i}, q_{b j}^{\dagger}\right]\right| \Phi_{0}^{M P}\right\rangle^{(0,2)} \\
&{ }^{e} O_{a i}^{(0,2)}=\left\langle\Phi_{0}^{M P}\left|\left[q_{a i}, \hat{O}\right]\right| \Phi_{0}^{M P}\right\rangle^{(0,2)} \\
&{ }^{d} \Pi_{a i b j}^{(1)}=\left\langle\Phi_{0}^{M P}\left|\left[\hat{P}, q_{a i} q_{b j}\right]\right| \Phi_{0}^{M P}\right\rangle^{(1)}=\left\langle\Phi_{0}^{M P}\left|\left[q_{a i}^{\dagger}, \hat{O}\right]\right| \Phi_{0}^{M P}\right\rangle^{(0,2)} \\
&{ }^{e} \Pi_{a i b j}^{(1)} P_{a i}^{(0,2)}=\left\langle\Phi_{0}^{M P}\left|\left[\hat{P}, q_{a i}^{\dagger} q_{b j}^{\dagger}\right]\right| \Phi_{0}^{M P}\right\rangle^{(1)} \\
&{ }^{e} P_{a i}^{(0,2)}=\left\langle\Phi_{0}^{M P}\left|\left[\hat{P}, q_{a i}^{\dagger}\right]\right| \Phi_{0}^{M P}\right\rangle^{(0,2)} \\
&{ }^{e} \Omega_{a i b j}^{(1)}\left.=\left\langle\Phi_{0}^{M P}\left|\left[q_{a i} q_{b j}, \hat{O}\right]\right| \Phi_{0}^{M P}\right\rangle^{(1)}, q_{a i}\right]\left|\Phi_{0}^{M P}\right\rangle^{(0,2)} \\
&{ }^{d}{ }_{a i b j}^{(1)}=\left\langle\Phi_{0}^{M P}\left|\left[q_{a i}^{\dagger} q_{b j}^{\dagger}, \hat{O}\right]\right| \Phi_{0}^{M P}\right\rangle^{(1)}
\end{aligned}
$$

where $\hat{H}$ is the field-free molecular electronic Hamiltonian of the system, $\Phi^{\mathrm{HF}}$ and $\Phi^{\mathrm{MP}}$ are the Hartree-Fock and Møller-Plesset perturbation theory wavefunctions and $q_{a i}^{\dagger}$ and $q_{a i}^{\dagger} q_{b j}^{\dagger}$ or $q_{a i}$ and $q_{a i} q_{b j}$ are respectively single and double second quantization excitation or deexcitation operators.

To evaluate the methods in this study reference polarizabilities were taken from the previous study ${ }^{4}$, where CC3 polarizabilities, for the same molecules and frequencies as in this work, were calculated with the aug-cc-pVTZ basis set. For a few values, it is not the 
true aug-cc-pVTZ value but the polarizability calculated with Sadlej's polarized triple zeta basis set, which was then corrected for the basis set effect in order to predict the aug-ccpVTZ polarizability. The predicted aug-cc-pVTZ polarizabilities were in the previous study statistically investigated and found to be reliable.<smiles>c1ccccc1</smiles>

Benzene<smiles>c1ccncc1</smiles>

Pyridine

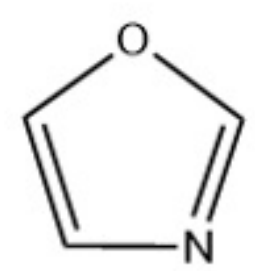

Oxazole<smiles>c1cncnc1</smiles>

\section{Pyrimidine}

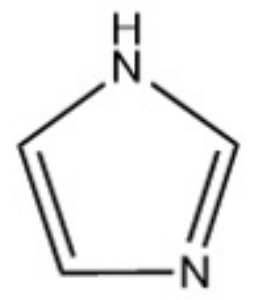

Imidazole<smiles>c1cnccn1</smiles>

Pyrazine

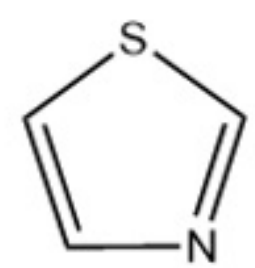

Thiazole<smiles>c1ccnnc1</smiles>

Pyridazine

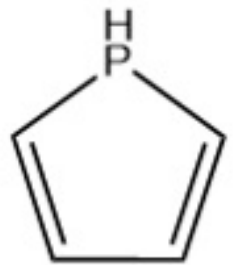

Phosphole<smiles>Oc1ccccc1</smiles>

Phenol<smiles>N#Cc1ccccc1</smiles>

Benzonitrile

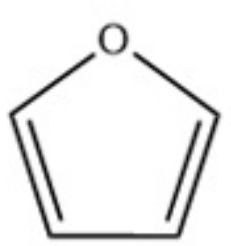

Furan

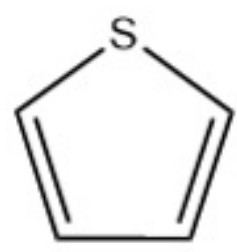

Thiophene

FIG. 1. Structures and names of molecules used in this study

\section{RESULTS AND DISCUSSION}

In the first section a quick investigation on the performance of Sadlej's polarized triple zeta basis set in comparison to Dunning's aug-cc-pVTZ basis set is made. In the following section the methods in this study will be tested against the CC3 reference values to benchmark their performance. Finally, in the last section the results of all methods will be compared with 
experimental polarizabilities.

\section{A. Basis set effects}

Previously the performance of different augmented Dunning basis sets was investigated in the calculation of static and dynamic polarizabilities for the same molecules at the SOPPA level ${ }^{4}$. It was found that at the SOPPA level there was no real gain in using the aug-cc-pVQZ basis set instead of the aug-cc-pVTZ, but that the computational time was significantly longer. For this set of molecules at least, there was neither found any difference when using the d-aug-cc-pVTZ basis set instead of the aug-cc-pVTZ basis set. Furthermore, the augcc-pVDZ results correlate slightly better with the aug-cc-pVTZ results than the results of calculations with the Sadlej's polarized triple zeta basis set. However, the slope of the linear regression of the results from Sadlej's basis set versus the aug-cc-pVTZ results was closer to one and thus reproduced the aug-cc-pVTZ values better than aug-cc-pVDZ. The previous study investigated the basis sets at the SOPPA level, where in this work we investigate methods which are approximations to SOPPA.

At the RPA level, one could use the fulfillment of the Thomas-Reiche-Kuhn ${ }^{52,53}$ (TRK) or similar sum rules as criterion for basis set completeness. However, previous calculations have shown that rather larger ${ }^{54}$ or specially optimized basis sets ${ }^{55,56}$ including both tight and diffuse functions are necessary to reach fulfillment of the TRK sum rule to two decimals, while for other properties such as polarizabilities one can reach sufficient basis set convergence with smaller and thus less complete basis sets and without particularly tight functions. ${ }^{4,57}$ One of the reasons for this is that the polarizability calculated as a sum over both bound and continuums excited states often converges much faster with the number of excited states than the TRK sum rule as e.g. recently illustrated for the hydrogen atom. ${ }^{58}$ This leads to weaker demands on the basis set. However, which incomplete basis sets are sufficiently converged for calculations of polarizabilities, can then become dependent on the employed computational method. For this reason it is of interest to see if the performance of Sadlej's basis set is affected, when lower-level methods are used. Therefore, we investigated in the present study the correlation between the aug-cc-pVTZ and Sadlej's basis set results for static and dynamic polarizabilities for all methods in this study, except the reference method, i.e. for SOPPA, RPA, HRPA, RPA(D) and HRPA(D). 
The results for the static and dynamic polarizabilities obtained with the different methods using Sadlej's and the aug-cc-pVTZ basis set can be found in Tables S1 to S3 of the supplemental material.

Table I shows the parameters of the linear regression of the correlation between the polarizabilities obtained with the two different basis sets following the form $y=a x+b$. The plots belonging to these correlations can be found in Figures S1 and S2 of the supplemental material. Though not much can be seen from the plots, they reveal that the two basis sets correlate, yielding comparable polarizabilities and that there is no clear tendency with respect to the deviations from the correlations. When looking at the dynamic cases compared to the static ones, one can see that these deviations are amplified. For the static case, in Table I, using all methods, the $R^{2}$ value is not quite satisfying. Furthermore, the offset $b$ is greater than 1 and the slope $a$ is not close enough to 1 for the correlation to be exact. Going to the dynamic case the $R^{2}$ becomes even smaller, the offset larger and the slope further away from 1. When comparing these correlations to the ones from the earlier study ${ }^{4}$, these correlations are clearly poorer. This suggests that the Sadlej's basis set is not as well performing with the RPA, HRPA, RPA(D) and HRPA(D) methods as for the SOPPA method alone.

TABLE I. Parameters in the linear regression $y=a x+b$ of static or frequency dependent polarizabilities (in au) calculated with Sadlej's basis set (y) versus the values obtained with the aug-cc-pVTZ basis set $(x)$

\begin{tabular}{|c|c|c|c|c|c|c|}
\hline \multirow[b]{2}{*}{ Methods included } & \multicolumn{3}{|c|}{ Static } & \multicolumn{3}{|c|}{ Dynamic } \\
\hline & $\mathbf{a}$ & b & $\mathbf{R}^{2}$ & $\mathbf{a}$ & $\mathbf{b}$ & $\mathbf{R}^{2}$ \\
\hline $\begin{array}{l}\text { SOPPA, RPA, HRPA, } \\
\text { RPA(D), HRPA(D) }\end{array}$ & 0.9922 & 1.0938 & 0.9979 & 0.9905 & 1.2120 & 0.9968 \\
\hline $\begin{array}{l}\text { RPA, HRPA, RPA(D), } \\
\operatorname{HRPA(D)}\end{array}$ & 0.9900 & 1.2213 & 0.9974 & 0.9872 & 1.3985 & 0.9961 \\
\hline
\end{tabular}

To further investigate the performance of Sadlej's basis set for the RPA, HRPA, RPA(D) and $\operatorname{HRPA}(\mathrm{D})$ methods, the correlation between the results with Sadlej's basis set and the aug-cc-pVTZ basis set is also investigated without including the SOPPA results. The difference from the previous case, including the SOPPA method, for both the static and 
dynamic case the correlations becomes poorer. The $R^{2}$ values are lower, the slopes are lower and the offsets are larger. This shows that the inclusion of the SOPPA method improves the correlation between the two basis sets. In the previous study, SOPPA was one of the lowest-level methods employed, where in this study it is the highest-level method beside the reference method, CC3. From this quick investigation it seems that the smaller basis set, Sadlej's basis set, in combination with these lower-level methods, no longer yield results of desired precision. In the rest of this study only the results with the aug-cc-pVTZ basis set will be further discussed.

TABLE II. Static polarizabilities (in au) calculated with the aug-cc-pVTZ basis set

\begin{tabular}{lccccccccccc}
\hline Molecules & $\mathbf{C C 3}^{a}$ & \multicolumn{2}{c}{ RPA } & \multicolumn{2}{c}{ HRPA } & \multicolumn{2}{c}{ SOPPA $^{a}$} & \multicolumn{3}{c}{ RPA(D) } & \multicolumn{2}{c}{ HRPA(D) } \\
& $\alpha$ & $\alpha$ & $D e v^{b}$ & $\alpha$ & $D e v^{b}$ & $\alpha$ & $D e v^{b}$ & $\alpha$ & $D e v^{b}$ & $\alpha$ & $D e v^{b}$ \\
\hline Benzene & 68.49 & 68.55 & 0.06 & 54.35 & -14.14 & 70.96 & 2.46 & 70.51 & 2.01 & 66.70 & -1.80 \\
Benzonitrile & 85.66 & 85.54 & -0.12 & 66.03 & -19.63 & 89.00 & 3.34 & 87.89 & 2.23 & 83.94 & -1.72 \\
Furan & 48.34 & 47.92 & -0.42 & 39.04 & -9.30 & 50.05 & 1.71 & 49.22 & 0.88 & 48.07 & -0.26 \\
Imidazole & 49.16 & 47.59 & -1.57 & 38.89 & -10.28 & 50.61 & 1.45 & 49.78 & 0.61 & 48.39 & -0.78 \\
Oxazole & 43.18 & 42.17 & -1.01 & 34.73 & -8.45 & 44.78 & 1.59 & 43.86 & 0.67 & 42.92 & -0.26 \\
Phenol & 74.15 & 72.53 & -1.62 & 76.48 & 2.33 & 77.59 & 3.44 & 58.17 & -15.98 & 73.41 & -0.74 \\
Pyrazine & 58.83 & 58.12 & -0.71 & 45.90 & -12.93 & 60.92 & 2.09 & 60.14 & 1.32 & 57.81 & -1.02 \\
Pyridazine & 58.73 & 57.73 & -1.00 & 45.88 & -12.86 & 60.79 & 2.06 & 60.01 & 1.28 & 57.77 & -0.96 \\
Pyridine & 63.19 & 62.72 & -0.47 & 50.01 & -13.18 & 65.79 & 2.60 & 65.14 & 1.95 & 62.70 & -0.49 \\
Pyrimidine & 57.95 & 56.48 & -1.48 & 45.76 & -12.20 & 60.64 & 2.69 & 59.82 & 1.86 & 57.61 & -0.35 \\
Pyrrol-N & 54.47 & 53.73 & -0.74 & 43.38 & -11.09 & 56.06 & 1.60 & 55.48 & 1.01 & 53.77 & -0.70 \\
Pyrrol-P & 73.52 & 72.93 & -0.58 & 57.81 & -15.71 & 76.06 & 2.54 & 75.42 & 1.90 & 72.64 & -0.88 \\
Thiazole & 58.87 & 57.14 & -1.72 & 46.78 & -12.08 & 61.24 & 2.37 & 60.04 & 1.17 & 58.49 & -0.37 \\
Thiophene & 63.85 & 63.05 & -0.80 & 51.07 & -12.78 & 66.07 & 2.22 & 65.30 & 1.45 & 63.43 & -0.42 \\
\hline
\end{tabular}

\section{B. Benchmarking of methods}

The results of all methods obtained with the aug-cc-pVTZ basis set have individually been compared to the CC3 results for static and dynamic polarizabilities. The calculated 
static and dynamic polarizabilities for the different methods can be found in Table II, IV and $\mathbf{V}$.

In Figure 2 a statistical analysis of the deviations from the CC3 results is shown for the static polarizabilities. One can immediately see that the results of the HRPA method deviate a lot from all the other results. They consistently underestimate the CC3 reference values. HRPA performs thus poorly, which is not really surprising as the method is known to overestimate electronic excitation energies ${ }^{7}$. On the other hand, the rest of the models perform somewhat similarly. The specific statistical values of their deviations from the reference values can be found in Table III.

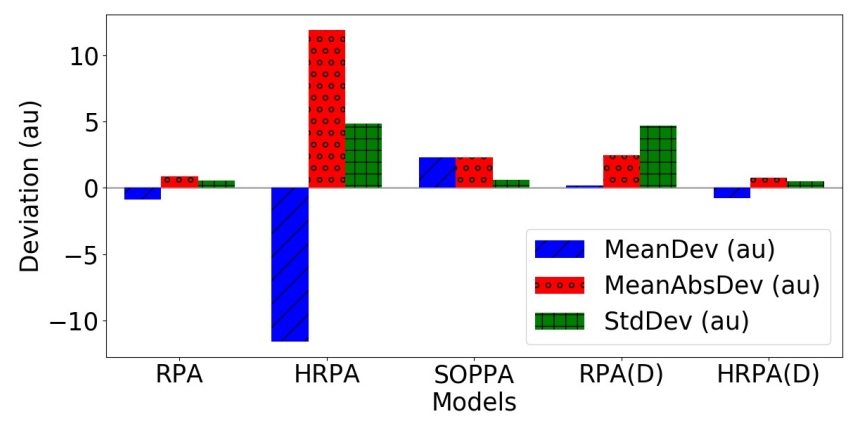

FIG. 2. Deviation from CC3 static polarizabilities

TABLE III. Deviations in static and dynamic polarizabilities (in au) of all methods with respect to the CC3 result with the aug-cc-pVTZ basis set

\section{Static Dynamic}

Method Mean Abs. Mean Std. Dev Mean Abs. Mean Std. Dev

\begin{tabular}{lcccccc}
\hline RPA & -0.87 & 0.88 & 0.56 & -0.93 & 0.95 & 0.66 \\
RPA(D) & 0.17 & 2.45 & 4.68 & 1.37 & 2.23 & 2.70 \\
HRPA & -11.59 & 11.59 & 4.48 & -14.66 & 14.66 & 3.51 \\
HRPA(D) & -0.77 & 0.77 & 0.49 & -1.50 & 1.50 & 0.76 \\
SOPPA & 2.30 & 2.30 & 0.61 & 2.97 & 2.97 & 0.86 \\
\hline
\end{tabular}

Comparing the RPA with its double corrected version, RPA(D), one can see that the mean deviation from the CC3 results is clearly reduced from -0.87 au to 0.17 au. RPA(D) has actually the smallest mean deviation among these methods. However, looking at the absolute mean deviations and the standard deviations of the errors, shows that RPA anyway 
performs better than RPA(D). While RPA almost consistently underestimates the CC3 results with a standard deviation of only $0.56 \mathrm{au}$, the errors of RPA(D) are spread around zero with an absolute mean deviation of 2.45 au. Furthermore, the standard deviation of RPA(D) is much higher, $4.68 \mathrm{au}$, meaning that this method is not very predictable in its deviations. As the RPA correlates closely with the CC3 performance, adding the doubles correction to RPA deteriorates the results, giving a greater deviation for the RPA(D) method from the CC3 results. The effect of adding the doubles corrections can in general be fairly large ${ }^{6,7}$, which is also illustrated here for polarizabilities calculated at the HRPA and HRPA(D) levels. The inclusion of the second-order contributions to the A, B and S matrices of RPA, leading to HRPA, results in a significant underestimation of the $\mathrm{CC} 3$ polarizabilities. This underestimation is undoubtedly improved by adding the double corrections, making HRPA(D) the method with the smallest mean deviation, $-0.77 \mathrm{au}$, from the CC3 results. HRPA(D) also turns out to be the most consistent method with the smallest standard deviation, $0.49 \mathrm{au}$. A similar drastic effect of the doubles correction on HRPA has previously been seen also for singlet and triplet excitation energies and NMR spin-spin coupling constants ${ }^{6,7}$. It is, however, quite surprising that both RPA and HRPA(D) perform better than SOPPA for the static polarizabilities of these molecules. SOPPA very consistently overestimates the polarizabilities compared to the CC3 results with a mean (absolute) deviation of 2.30 au and a standard deviation of $0.61 \mathrm{au}$. This is in contrast to previous findings for smaller molecules or atoms ${ }^{36,59-62}$. On the other hand, previous studies of vertical excitation energies for some of the same molecules showed that SOPPA consistently underestimates excitation energies to singlet states by roughly $0.5 \mathrm{eV}^{7,23}$, which then should lead to an overestimation of polarizabilities. Consequently, it is more surprising that RPA gives such a good agreement with the high-level CC3 results for the polarizabilities of these molecules. SOPPA as an only second-order method overestimates then the remaining, rather small electron correlation correction.

Turning now to the dynamic case, the statistical analysis in Figure $\mathbf{3}$ shows a similar trend as for the static case with only minor differences. The numerical values for the mean deviations and standard deviations are also shown in Table III. In general the mean deviations from the $\mathrm{CC} 3$ results are larger for all methods. But the change is smallest for RPA, which thus is the method predicting dynamic polarizabilities closest and most consistently to the CC3 results with a mean deviation of -0.93 au and a standard deviation 
TABLE IV. Dynamic polarizabilities (in au) at wavelength: $632 \mathrm{~nm}$, calculated with the aug-ccpVTZ basis set

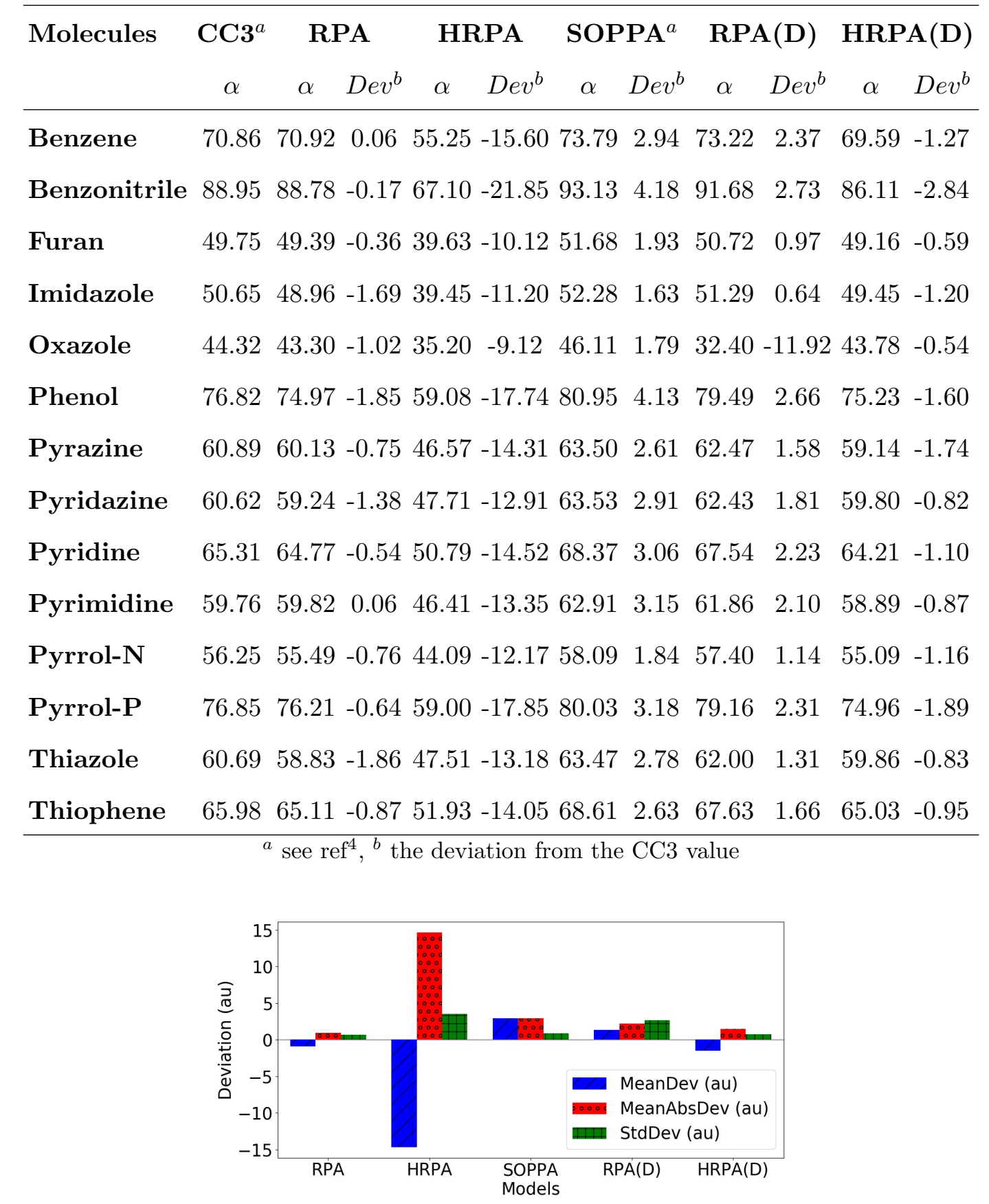

FIG. 3. Deviation from CC3 dynamic polarizabilities

of $0.66 \mathrm{au}$. RPA performs thus better than HRPA(D) in contrast to the static polarizabilities and both perform better than SOPPA. The mean deviation of the SOPPA results is almost twice as large as for HRPA(D) and the standard deviation is with 0.86 au also slightly 
TABLE V. Dynamic polarizabilities (in au) at wavelength: $488 \mathrm{~nm}$, calculated with the aug-ccpVTZ basis set

\begin{tabular}{|c|c|c|c|c|c|c|c|c|c|c|c|}
\hline \multirow[t]{2}{*}{ Molecules } & \multirow{2}{*}{$\begin{array}{c}\mathbf{C C 3}^{a} \\
\alpha\end{array}$} & \multicolumn{2}{|c|}{ RPA } & \multicolumn{2}{|c|}{ HRPA } & \multicolumn{2}{|c|}{$\operatorname{SOPPA}^{a}$} & \multicolumn{2}{|c|}{ RPA(D) } & \multicolumn{2}{|c|}{ HRPA(D) } \\
\hline & & $\alpha$ & $D e v^{b}$ & $\alpha$ & $D e v^{b}$ & $\alpha$ & $D e v^{b}$ & $\alpha$ & $D e v^{b}$ & $\alpha$ & $D e v^{b}$ \\
\hline Benzene & 72.63 & 72.68 & 0.06 & 55.89 & -16.74 & 75.96 & 3.34 & 75.28 & 2.65 & 70.83 & -1.79 \\
\hline Benzonitrile & 91.46 & 91.25 & -0.21 & 67.85 & -23.60 & 96.40 & 4.94 & 94.61 & 3.15 & 87.66 & -3.79 \\
\hline Furan & 50.80 & 50.50 & -0.30 & 40.05 & -10.75 & 52.92 & 2.12 & 51.84 & 1.03 & 49.93 & -0.87 \\
\hline Imidazole & 51.76 & 49.98 & -1.78 & 39.85 & -11.91 & 53.54 & 1.79 & 52.42 & 0.66 & 50.22 & -1.54 \\
\hline Oxazole & 45.16 & 44.14 & -1.02 & 35.53 & -9.63 & 47.10 & 1.94 & 45.92 & 0.76 & 44.40 & -0.76 \\
\hline Phenol & 78.86 & 76.81 & -2.05 & 59.73 & -19.13 & 83.61 & 4.75 & 81.79 & 2.93 & 76.52 & -2.33 \\
\hline Pyrazine & 62.50 & 61.69 & -0.81 & 47.05 & -15.45 & 65.65 & 3.15 & 64.32 & 1.81 & 60.10 & -2.40 \\
\hline Pyridazine & 62.07 & 60.55 & -1.51 & 48.21 & -13.86 & 65.50 & 3.43 & 64.05 & 1.98 & 60.78 & -1.29 \\
\hline Pyridine & 66.91 & 66.31 & -0.60 & 51.33 & -15.58 & 70.36 & 3.45 & 69.37 & 2.46 & 65.28 & -1.63 \\
\hline Pyrimidine & 61.11 & 59.34 & -1.77 & 46.86 & -14.25 & 64.68 & 3.57 & 63.40 & 2.28 & 59.80 & -1.32 \\
\hline Pyrrol-N & 57.59 & 56.83 & -0.77 & 44.58 & -13.01 & 59.64 & 2.04 & 58.84 & 1.24 & 56.05 & -1.55 \\
\hline Pyrrol-P & 79.51 & 78.81 & -0.71 & 59.86 & -19.66 & 83.32 & 3.80 & 82.19 & 2.68 & 76.66 & -2.85 \\
\hline Thiazole & 62.05 & 60.09 & -1.97 & 48.02 & -14.03 & 65.18 & 3.13 & 63.47 & 1.41 & 60.84 & -1.21 \\
\hline Thiophene & 67.58 & 66.65 & -0.93 & 52.53 & -15.04 & 70.57 & 2.99 & 69.40 & 1.82 & 66.18 & -1.40 \\
\hline
\end{tabular}

larger for SOPPA than for HRPA(D) with 0.76 au. Equal to the static polarizabilities, the results obtained with the HRPA model are very different from the CC3 results. And also the addition of the doubles corrections to RPA, leading to RPA(D), diminishes again its performance. The mean deviation of the RPA(D) is still smaller than for the other methods apart from RPA, but the mean absolute deviation and in particular the standard deviation is again significantly larger.

To shed some light on this maybe unexpected good performance of RPA in calculations of polarizabilities for these molecules in clear contrast to smaller molecules, ${ }^{36,59-62}$ it is useful to remember the sum-over-states expression for the static polarizability in a finite basis set in atomic units

$$
\alpha=\sum_{n \neq 0}\left(E_{n}-E_{0}\right)^{-2} f_{n 0}^{L}
$$


where $f_{n 0}^{L}$ is the oscillator strength of the excitation from the ground state with energy $E_{0}$ to the excited state with energy $E_{n}$, and the expression for the TRK sum rule $S^{L}(0)$

$$
S^{L}(0)=\sum_{n \neq 0} f_{n 0}^{L}
$$

which for exact oscillator strengths is equal to the number of electrons in the system. At the RPA level and for a complete basis set the TRK sum rule is also fulfilled, ${ }^{63,64}$ which means that the sum of all corrections from higher level methods must be zero. However, the individual terms in a perturbation theory expansion of the TRK sum rule do not vanish individually, implying e.g. that TRK sum rules evaluated at the SOPPA or CCSD level will no longer be equal to the number of electrons. ${ }^{54,65}$ One could therefore argue, that a similar effect is at work here for the polarizabilities of these molecules, leading to an overestimation of correlation contributions at low orders of perturbation theory.

Another way to look at it is based on the observation that HRPA(D) is slightly better than RPA for the static case and slightly worse for the dynamic case, while SOPPA is somewhat but consistently worse than both methods and HRPA is very much worse than all three methods. This signifies that doubles excitations are certainly necessary to balance the second-order single excitation terms included in HRPA, but including them in an iterative way as in SOPPA leads to an overestimation of the correlation correction, while the noniterative treatment in $\operatorname{HRPA}(\mathrm{D})$ leads to better results, although not really better than the simple RPA results. One should also remember here that CC2, another second order method with iterative doubles contributions, was shown to perform even worse than SOPPA for the same property and molecules, ${ }^{4}$ while CCSD gave the best agreement with the CC3 results with errors of about one third of the size of the errors of RPA and thus clearly better than RPA. The difference between CCSD and CC2 is in the expressions for the contributions from doubles excitations, which are approximated in $\mathrm{CC} 2$, indicating again, that is the correct or balanced treatment of these double excitation contributions which is of importance for a good description of the correlation corrections to the polarizabilities of these molecules.

The good performance of RPA and HRPA(D) justifies thus to prefer them for calculations of polarizabilities of these molecules over the more computationally demanding SOPPA method. The usage of the these "simpler" methods instead of the larger and more computationally demanding methods, opens up for the possibility of going to larger aromatic molecules or using larger basis sets as possible with SOPPA. Taking the static polarizability 
of benzene, as a classic example, the total CPU time used in Dalton16 on one CPU for a RPA calculation with the aug-cc-pVTZ basis set was 19 minutes and 27 seconds, whereas the same calculation at the HRPA(D) level took 44 minutes and 10 seconds on the same computer and the SOPPA calculation lasted 1 hour 6 minutes and 59 seconds. This difference in CPU time becomes only larger for larger and less symmetrical molecules, emphasizing the potential of the lower-level methods.

\section{Comparison with experiment}

Benchmarking computational methods against experimental values can be an alternative criteria of performance of the methods. This can show how good a method is at reproducing experimental values though theoretically predicted values and experimental ones are not truly based on the same assumptions. This comparison is not without problems, because experimental values are often measured in solvents and always for a particular temperature. For an optimal comparison this would require to include solvent, ${ }^{66,67}$ vibrational averaging and temperature effects ${ }^{68,69}$ also in the calculations. None of these were, however, included in our calculations presented here. Nevertheless, we will in this section attempt to make such a comparison with experimental values for the molecules where values could be found in the literature.

Table VI shows these experimental values for static polarizabilities and the absolute deviations from them for each of the methods used in this study as well as the mean absolute deviations and standard deviations. Not enough experimental values for the dynamic polarizabilities were found to justify an analysis. As expected, the high-level coupled cluster method CC3 reproduces the experimental values with the lowest absolute mean deviation and a small standard deviation. Although RPA was found to perform better than RPA(D) when comparing to the CC3 results, RPA(D) and also HRPA(D) reproduce the experimental values better than RPA. RPA(D) performs on average also better than HRPA(D) and performs actually almost as good as CC3. But it is interesting to see that for the individual molecules there are quite large differences between the two methods. RPA(D) exhibits e.g. only small errors for thiophene, pyrimidine and pyrazine, for which CC3 shows larger errors. HRPA is again found to be incomparable with any of the other methods and exhibits errors relative to experiment, which are an order of magnitude larger than for some of the 
other methods. SOPPA performs on average slightly worse than RPA but is more consistent in its deviations form the experimental values. Summarizing, one can say that for static polarizabilities the performance of HRPA and SOPPA is almost the same when comparing with experimental values or with the high-level CC3 results, but there are differences with respect to the performance of the other three methods RPA, RPA(D) and HRPA(D), which however is not due to the smaller test set in the comparison with the experimental values.

TABLE VI. Static polarizabilites (in au): absolute deviations from experimental values. The polarizabilities are calculated with the aug-cc-pVTZ basis set.

\begin{tabular}{|c|c|c|c|c|c|c|c|}
\hline Molecule & Exp. Val. & RPA & HRPA & SOPPA & RPA(D) & $\operatorname{RPA}(\mathrm{D})$ & CC3 \\
\hline & & this & work & Ref. 4 & thi & ork & Ref. 4 \\
\hline Benzene & $67.48^{a}$ & 1.07 & 13.13 & 3.48 & 3.03 & 0.78 & 1.01 \\
\hline Furan & $48.59^{c}$ & 0.67 & 9.55 & 1.46 & 0.63 & 0.52 & 0.25 \\
\hline Pyrazine & $60.62^{d}$ & 2.50 & 14.72 & 0.30 & 0.48 & 2.81 & 1.79 \\
\hline Pyridazine & $59.32^{d}$ & 1.59 & 13.44 & 1.47 & 0.69 & 1.55 & 0.59 \\
\hline Pyridine & $64.11^{d}$ & 1.39 & 14.10 & 1.68 & 1.03 & 1.41 & 0.92 \\
\hline Pyrimidine & $59.35^{d}$ & 2.87 & 13.59 & 1.29 & 0.47 & 1.74 & 1.54 \\
\hline Pyrrole & $53.47^{b}$ & 0.26 & 10.09 & 2.59 & 2.01 & 0.30 & 1.00 \\
\hline Thiophene & $65.18^{c}$ & 2.13 & 14.11 & 0.89 & 0.12 & 1.75 & 1.33 \\
\hline Abs. Mean Dev. & & 1.56 & 12.84 & 1.65 & 1.06 & 1.36 & 1.05 \\
\hline Standard Dev. & & 1.37 & 1.93 & 0.99 & 1.11 & 0.94 & 1.08 \\
\hline
\end{tabular}

\section{CONCLUSION}

For 14 (hetero-)aromatic molecules static and dynamic polarizabilities calculated at the RPA, RPA(D), HRPA, HRPA(D) and SOPPA level using the aug-cc-pVTZ basis set were compared to $\mathrm{CC} 3$ reference values and experiment.

The benchmark study gave unexpected results as RPA, for both static and dynamic frequencies, was found to give results in better agreement with the CC3 results than SOPPA. HRPA(D) was found to perform closest to the CC3 values for static polarizabilities, while for dynamic polarizabilities it was RPA. For both cases the RPA(D) and SOPPA method 
performed similarly. HRPA, finally, was in both the static and dynamic case found to lead to errors an order of magnitude larger than the other methods. The observation that RPA outperforms SOPPA in calculations of polarizabilities has previously not been reported in the literature and rises the interest in further studies on other organic molecules.

The comparison to the limited number of experimental values showed more or less the same for static polarizabilities, with the exception of RPA(D) now reproducing the experimental values better than RPA.

Independent of calculating static or dynamic polarizabilities, this study found that the RPA and HRPA(D) methods would yield results comparable with CC3 results, at least for these kinds of hetero-aromatic molecules. Assuming that this is not just a consequence of this specific set of molecules, the lower level methods, RPA and HRPA(D), would allow for calculations on much larger systems as with SOPPA and in particular CC3. For reproducing experimental values the RPA and HRPA(D) methods are still found to be adequate and better than SOPPA for the investigated set of molecules.

Comparing the static and dynamic polarizabilities calculated with the aug-cc-pVTZ basis set and with Sadlej's polarized triple zeta basis set showed larger discrepancies for the polarization propagator methods studied in this work as were found previously for e.g. coupled cluster methods. Sadlej's basis set was thus not able to perfectly reproduce the aug-cc-pVTZ results apart from a constant offset for these molecules and methods.

\section{SUPPLEMENTARY MATERIAL}

Table S1 lists static polarizabilities for the methods: RPA, HRPA, RPA(D) and HRPA(D) using the basis sets: aug-cc-pVTZ and Sadlej-pVTZ. Table S2 and S3 lists the dynamic polarizabilities at $632 \mathrm{~nm}$ and $488 \mathrm{~nm}$ respectively for the same methods and basis sets. Figures $\mathbf{S} 1$ and $\mathbf{S} 2$ depicts the correlations found in Table I.

\section{ACKNOWLEDGMENTS}

The Department of Chemistry, University of Copenhagen, is thanked for providing access to its high-performance computer cluster. Christoffer H. S. Møller is gratefully acknowledged for providing the python code for all graphs in this work and for adapting the code to the 
wishes of the authors.

\section{DATA AVAILABILITY STATEMENT}

The data that supports the findings of this study are available within the article [and its supplementary material]. Further data are available from the corresponding author upon reasonable request.

\section{REFERENCES}

${ }^{1}$ O. Christiansen, J. Gauss, and J. F. Stanton, Chem. Phys. Lett. 292, 437 (1998).

${ }^{2}$ J. Rezac, L. Simova, and P. Hobza, J. Chem. Theory Comput. 9, 364 (2013).

${ }^{3}$ P.-F. Loos, F. Lipparini, M. Boggio-Pasqua, A. Scemama, and D. Jacquemin, J. Chem. Theory Comput. 16, 1711 (2020).

${ }^{4}$ M. W. Jørgensen, R. Faber, A. Ligabue, and S. P. A. Sauer, J. Chem. Theory Comput. 16, 3006 (2020).

${ }^{5}$ O. Christiansen, K. L. Bak, H. Koch, and S. P. A. Sauer, Chem. Phys. Lett. 284, 47 (1998).

${ }^{6}$ A. K. Schnack-Petersen, P. A. B. Haase, R. Faber, P. F. Provasi, and S. P. A. Sauer, J. Comp. Chem. 39, 2647 (2018).

${ }^{7}$ P. A. B. Haase, R. Faber, P. F. Provasi, and S. P. A. Sauer, J. Comp. Chem. 41, 43 (2019).

${ }^{8}$ D. J. Rowe, Rev. Mod. Phys. 40, 153 (1968).

${ }^{9}$ T.-I. Shibuya and V. McKoy, Phys. Rev. A. 2, 2208 (1970).

${ }^{10}$ E. S. Nielsen, P. Jørgensen, and J. Oddershede, J. Chem. Phys. 73, 6238 (1980).

${ }^{11}$ M. J. Packer, E. K. Dalskov, T. Enevoldsen, H. J. A. Jensen, and J. Oddershede, J. Chem. Phys. 105, 5886 (1996).

${ }^{12}$ K. L. Bak, H. Koch, J. Oddershede, O. Christiansen, and S. P. A. Sauer, J. Chem. Phys. 112, $4173(2000)$.

${ }^{13}$ P. F. Provasi, G. A. Aucar, and S. P. A. Sauer, J. Chem. Phys. 115, 1324 (2001).

${ }^{14}$ P. F. Provasi, G. A. Aucar, M. Sanchez, I. Alkorta, J. Elguero, and S. P. A. Sauer, J. Phys. Chem. A 109, 6555 (2005). 
${ }^{15}$ H. Kjær, S. P. A. Sauer, and J. Kongsted, J. Chem. Phys. 133, 144106 (2010).

${ }^{16}$ H. Kjær, S. P. A. Sauer, J. Kongsted, Y. Y. Rusakov, and L. B. Krivdin, Chem. Phys. 381, 35 (2011).

${ }^{17}$ P.-O. Åstrand, P. S. Ramanujam, S. Hvilsted, K. L. Bak, and S. P. A. Sauer, J. Am. Chem. Soc. 122, 3482 (2000).

${ }^{18}$ P.-O. Åstrand, P. Sommer-Larsen, S. Hvilsted, P. S. Ramanujam, K. L. Bak, and S. P. A. Sauer, Chem. Phys. Lett. 325, 115 (2000).

${ }^{19}$ P.-O. Åstrand, K. L. Bak, and S. P. A. Sauer, Chem. Phys. Lett. 343, 171 (2001).

${ }^{20}$ H. H. Falden, K. R. Falster-Hansen, K. L. Bak, S. Rettrup, and S. P. A. Sauer, J. Phys. Chem. A 113, 11995 (2009).

${ }^{21}$ M. Schreiber, M. R. Silva-Junior, S. P. A. Sauer, and W. Thiel, J. Chem. Phys. 128, 134110 (2008).

${ }^{22}$ S. P. A. Sauer, M. Schreiber, M. R. Silva-Junior, and W. Thiel, J. Chem. Theory Comput. 5, 555 (2009).

${ }^{23}$ S. P. A. Sauer, H. F. Pitzner-Frydendahl, M. Buse, H. J. A. Jensen, and W. Thiel, Mol. Phys. 113, 2026 (2015).

${ }^{24}$ C. H. S. Møller, A. K. Schnack-Petersen, and S. P. A. Sauer, Mol. Phys. , DOI:10.1080/00268976.2020.1757773 (2020).

${ }^{25}$ J. Schirmer, Phys. Rev. A 26, 2395 (1982).

${ }^{26}$ J. Schirmer and F. Mertins, J. Phys. B: At. Mol. Opt. Phys. 29, 3559 (1996).

${ }^{27}$ A. B. Trofimov and J. Schirmer, J. Phys. B: At. Mol. Opt. Phys. 28, 2299 (1995).

${ }^{28}$ A. B. Trofimov, I. L. Krivdina, J. Weller, and J. Schirmer, Chem. Phys. 329, 1 (2006).

${ }^{29}$ J. Geertsen, S. Eriksen, and J. Oddershede, Adv. Quantum Chem. 22, 167 (1991).

${ }^{30}$ J. Scirmer, A. B. Trofimov, and G. Stelter, J. Chem. Phys. 109, 4734 (1998).

${ }^{31}$ A. B. Trofimov, G. Stelter, and J. Schirmer, J. Chem. Phys. 111, 9982 (1999).

${ }^{32}$ S. Knippenberg, D. R. Rehn, M. Wormit, J. H. Starcke, I. L. Rusakova, A. B. Trofimov, and A. Dreuw, J. Chem. Phys. 136, 064107 (2012).

${ }^{33}$ T. Fransson, D. R. Rehn, A. Dreuw, and P. Norman, J. Chem. Phys. 146, 094301 (2017).

${ }^{34}$ J. Geertsen and J. Oddershede, J. Chem. Phys. 85, 2112 (1986).

${ }^{35}$ S. P. A. Sauer, J. Phys. B: At. Mol. Opt. Phys. 30, 3773 (1997).

${ }^{36}$ S. P. A. Sauer and J. Oddershede, Int. J. Quantum Chem. 50, 317 (1994).

${ }^{37}$ I. Paidarová and S. P. A. Sauer, Adv. Quantum Chem. 48, 185 (2005). 
${ }^{38}$ S. P. A. Sauer and I. Paidarová, Computing Letters 3, 399 (2007).

${ }^{39}$ I. Paidarová and S. P. A. Sauer, Collect. Czech. Chem. Commun. 73, 1415 (2008).

${ }^{40}$ M. Hodecker, A. L. Dempwolff, D. R. Rehn, P. Norman, and A. Dreuw, J. Phys. Chem. 150, 174104 (2019).

${ }^{41}$ M. Hodecker, D. R. Rehn, P. Norman, and A. Dreuw, J. Phys. Chem. 150, 174105 (2019). ${ }^{42}$ T. H. Dunning, J. Chem. Phys. 90, 1007 (1989).

${ }^{43}$ R. A. Kendall, T. H. Dunning, and R. J. Harrison, J. Chem. Phys. 96, 6796 (1992).

${ }^{44}$ D. E. Woon and T. H. Dunning, J. Chem. Phys. 98, 1358 (1993).

${ }^{45}$ A. J. Sadlej, Collect. Czech. Chem. Commun. 53, 1995 (1988).

${ }^{46}$ A. J. Sadlej, Theor. Chim. Acta. 81, 339 (1992).

${ }^{47}$ D. J. Rowe, Rev. Mod. Phys. 40, 153 (1968).

${ }^{48}$ T.-I. Shibuya and V. McKoy, Phys. Rev. A 2, 2208 (1970).

${ }^{49}$ K. Aidas, C. Angeli, K. L. Bak, V. Bakken, R. Bast, L. Boman, O. Christiansen, R. Cimiraglia, S. Coriani, P. Dahle, E. K. Dalskov, U. Ekström, T. Enevoldsen, J. J. Eriksen, P. Ettenhuber, B. Fernández, L. Ferrighi, H. Fliegl, L. Frediani, K. Hald, A. Halkier, C. Hättig, H. Heiberg, T. Helgaker, A. C. Hennum, H. Hettema, E. Hjertenæs, S. Høst, I.-M. Høyvik, M. F. Iozzi, B. Jansik, H. J. A. Jensen, D. Jonsson, P. Jørgensen, J. Kauczor, S. Kirpekar, T. Kjærgaard, W. Klopper, S. Knecht, R. Kobayashi, H. Koch, J. Kongsted, A. Krapp, K. Kristensen, A. Ligabue, O. B. Lutnæs, J. I. Melo, K. V. Mikkelsen, R. H. Myhre, C. Neiss, C. B. Nielsen, P. Norman, J. Olsen, J. M. H. Olsen, A. Osted, M. J. Packer, F. Pawlowski, T. B. Pedersen, P. F. Provasi, S. Reine, Z. Rinkevicius, T. A. Ruden, K. Ruud, V. Rybkin, P. Salek, C. C. M. Samson, A. S. de Merás, T. Saue, S. P. A. Sauer, B. Schimmelpfennig, K. Sneskov, A. H. Steindal, K. O. Sylvester-Hvid, P. R. Taylor, A. M. Teale, E. I. Tellgren, D. P. Tew, A. J. Thorvaldsen, L. Thøgersen, O. Vahtras, M. A. Watson, D. J. D. Wilson, M. Ziolkowski, and H. Ågren, WIREs Comput. Mol. Sci. 4, 269 (2014).

${ }^{50}$ O. Christiansen, K. L. Bak, H. Koch, and S. P. A. Sauer, Chem. Phys. Lett. 284, 47 (1998).

${ }^{51}$ E. S. Nielsen, P. Jørgensen, and J. Oddershede, J.Chem. Phys. 73, 6238 (1980).

${ }^{52}$ W. Kuhn, Z. Physik 33, 408 (1925).

${ }^{53} \mathrm{~F}$. Reiche and W. Thomas, 34, 510 (1925). 
${ }^{54}$ S. P. A. Sauer, I. Ul Haq, J. R. Sabin, J. Oddershede, O. Christiansen, and S. Coriani, Mol. Phys. 112, 751 (2014).

${ }^{55}$ S. P. A. Sauer, J. Oddershede, and J. R. Sabin, J. Phys. Chem. A 110, 8811 (2006).

${ }^{56}$ S. Bruun-Ghalbia, S. P. A. Sauer, J. Oddershede, and J. R. Sabin, J. Phys. Chem. B 114, 633 (2010).

${ }^{57}$ T. B. Pedersen, A. M. J. Sánchez de Merás, and H. Koch, J. Chem. Phys. 120, 8887 (2004).

${ }^{58}$ R. Cabrera-Trujillo, S. P. A. Sauer, J. R. Sabin, and J. Oddershede, Adv. Quantum Chem. 80, 127 (2019).

${ }^{59}$ S. P. A. Sauer, G. H. F. Diercksen, and J. Oddershede, Int. J. Quantum Chem. 39, 667 (1991).

${ }^{60}$ M. J. Packer, E. K. Dalskov, S. P. A. Sauer, and J. Oddershede, Theor. Chim. Acta 89, 323 (1994).

${ }^{61}$ S. P. A. Sauer, J. Phys. B: At. Mol. Opt. Phys. 30, 3773 (1997).

${ }^{62}$ E. K. Dalskov and S. P. A. Sauer, J. Phys. Chem. A 102, 5269 (1998).

${ }^{63}$ P. Jørgensen and J. Oddershede, J. Chem. Phys. 78, 1898 (1983).

${ }^{64}$ J. Schirmer, Many-Body Methods for Atoms, Molecules and Clusters (Springer Nature Switzerland, Cham, 2018) Chap. 15.

${ }^{65}$ J. Oddershede and J. R. Sabin, Phys. Rev. A 39, 5565 (1989).

${ }^{66}$ A. Møgelhøj, K. Aidas, K. V. Mikkelsen, S. P. A. Sauer, and J. Kongsted, J. Chem. Phys. 130, 134508 (2009).

${ }^{67}$ K. Ruud, L. Frediani, R. Cammi, and B. Mennucci, Int. J. Mol. Sci. 4, 119 (2003).

${ }^{68}$ T. A. Ruden and K. Ruud, in Calculation of NMR and EPR Parameters: Theory and Applications, edited by M. Kaupp, V. G. Malkin, and M. Bühl (Wiley-VCH, Weinheim, 2004) Chap. 10, pp. 153-173.

${ }^{69}$ R. Faber, J. Kaminsky, and S. P. A. Sauer, in Gas Phase NMR, edited by K. Jackowski and M. Jaszunski (Royal Society of Chemistry, London, 2016) Chap. 7, pp. 219-268.

${ }^{70}$ O. Christiansen, C. Hättig, and P. Jørgensen, Spectrochim acta A 55, 509 (1999).

${ }^{71}$ A. Hinchliffe and H. J. Soscún M., J. Mol. Struct 331, 109 (1995).

${ }^{72}$ K. Kamanda, M. Ueda, H. Nagao, K. Tawa, T. Sugino, Y. Shmizu, and K. Ohta, J. Phys. Chem. 104, 4723 (2000). 
${ }^{73}$ H. Soscún, Y. Bermúdez, O. Castellano, and J. Hernández, Chem. Phys. Lett. 396, 117 (2004). 


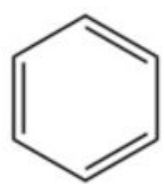

Benzene
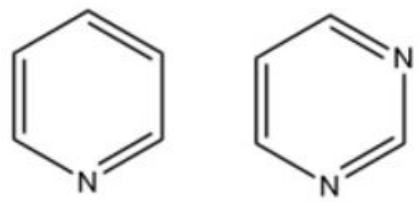

Pyridine

Oxazole
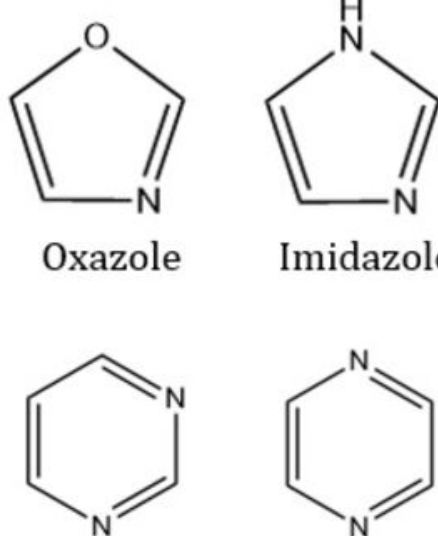

Pyrazine

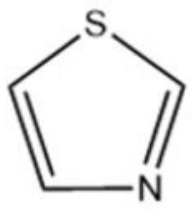

Thiazole

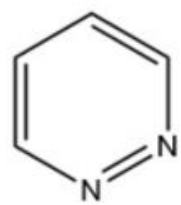

Pyridazine

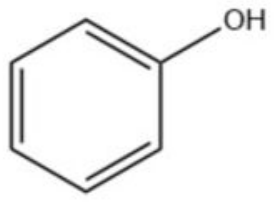

Phenol

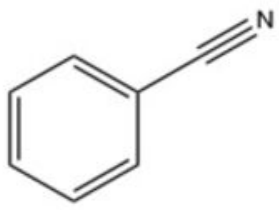

Benzonitrile

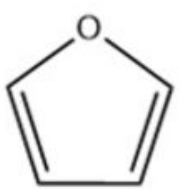

Furan

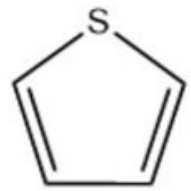

Thiophene

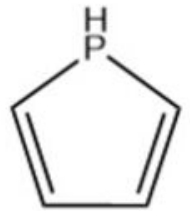

Phosphole

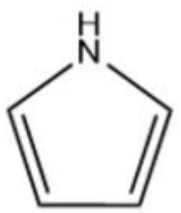

Pyrrole 


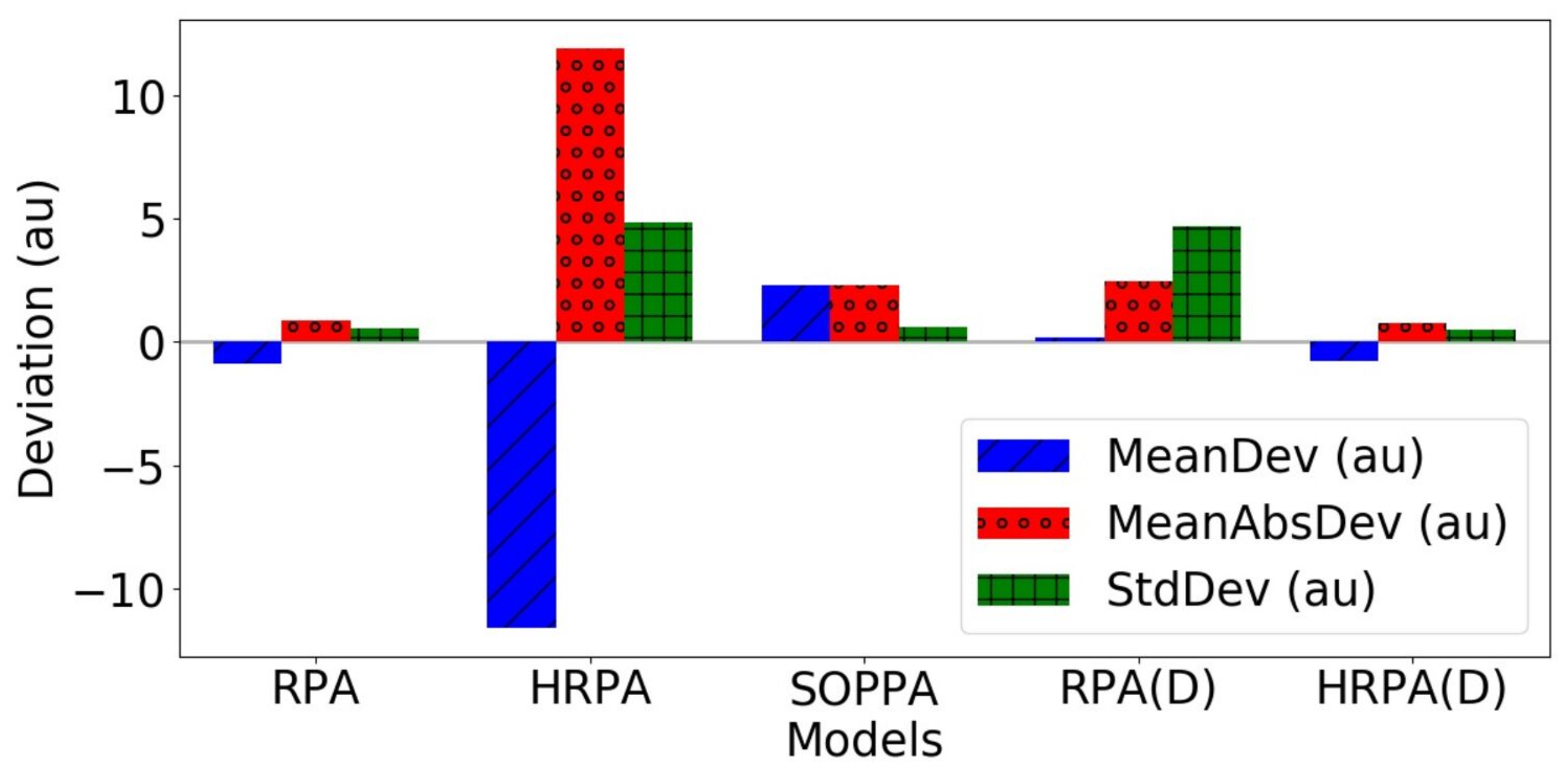




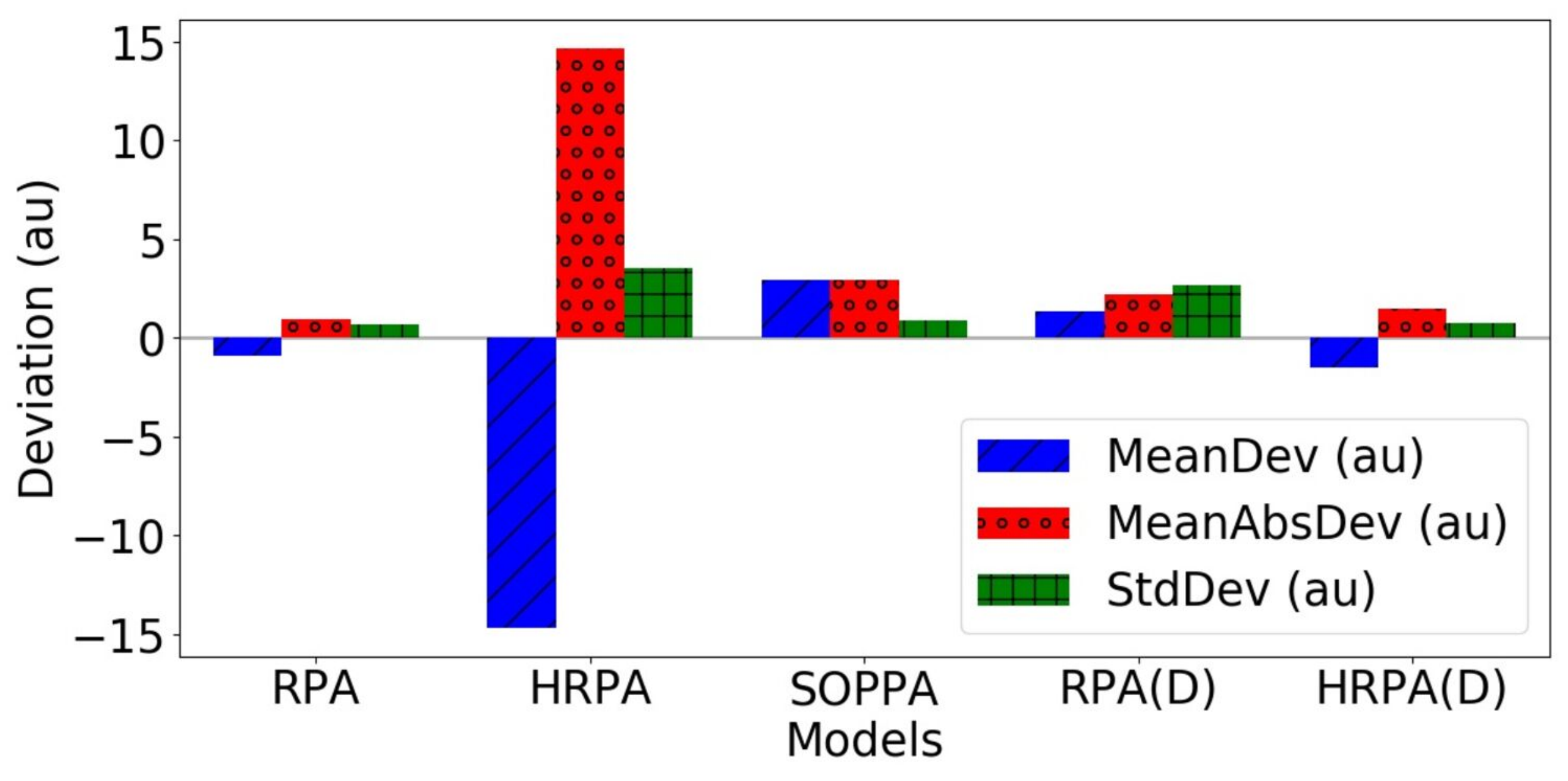

